\title{
$\mathscr{N}=2$ SUSY and Representation Theory: An Introduction
}

\section{Sergio Cecotti*}

SISSA, via Bonomea 265, I-33100 Trieste, Italy

E-mail: cecotti@sissa.it

The determination of the exact BPS spectrum of a (large class of) four-dimensional $\mathscr{N}=2$ QFT's is equivalent to the Representation Theory of certain associative algebras, namely the path algebras of quivers bounded by the Jacobian ideal of a superpotential $\mathscr{W}$. In this contest there is a detailed dictionary between QFT and Representation Theory. Properties which are elementary on one side are tipically subtle and hard to prove in the other. Hence both subjects have something to learn from the correspondence.

7th International Conference on Mathematical Methods in Physics, CBPF, Rio de Janeiro,

April 16-20, 2012

* Speaker. 


\section{Introduction}

A fundamental problem in high energy physics is the computation of non-perturbative quantities in four-dimensional non-Abelian gauge theories coupled to arbitrary matter. Unfortunately, the present state of the art leads to exact computations only if the gauge theory has enough supersymmetry. In this respect $\mathscr{N}=2$ supersymmetry is a natural laboratory in which the low-energy physics may be exactly computed [1,2].

The main question in the non-perturbative physics of $4 \mathrm{~d} \mathscr{N}=2$ QFT's is the determination of the spectrum of BPS particles as a function of the various parameters of the theory [3]-[22]. From refs. [23]- [30] it follows that there is a large class of $4 \mathrm{~d} \mathscr{N}=2$ QFT's ('almost all') for which the computation of the BPS mass spectrum is mapped into the Representation Theory (RT) of associative algebras.

This map is however quite peculiar. Virtually all (non-perturbative) aspects of the QFT can be rephrased in the language of algebras [28]; it appears that we have a complete dictionary between the two subjects. What is peculiar, is that the map works like a kind of 'trivial/deep duality' in the sense that, using the dictionary, very elementary QFT statements will map into quite deep and sophisticated results in RT, some of which — for the very simplest QFT's — are known theorems in mathematics which require full books to be proven, while dually totally trivial RT statements get mapped into quite deep non-perturbative results in the QFT side. Thus we can generate many deep results in both subjects just by applying the dictionary to the trivial results of the other one.

The purpose of this introductory is to describe the $\mathscr{N}=2$ QFT $\leftrightarrow$ RT correspondence and give some general consequence. More details may be found in the references.

This note covers part of the material presented at the 7th International Conference on Mathematical Methods in Physics at CBPF in Rio de Janeiro, April 16-20, 2012. I thank the organizers for the invitation.

\section{Associative Algebras}

Let us start by reviewing the RT side. The relevant algebras are associative, unital, and defined over an algebraically closed field which we take to be $\mathbb{C}$. Most of the mathematical statements require the algebra to be finite-dimensional; although it is not true that all algebras relevant for $\mathscr{N}=2$ QFT's are finite-dimensional, for the physical questions tipically they behave as they were finite-dimensional.

Given such an algebra $\mathscr{A}$, one is interested in its representations, that is, in its left (or right) modules which form an Abelian category $\bmod \mathscr{A}$. A general module is the direct sum of indecomposable ones, hence it suffices to study the indecomposable modules up to isomorphism. The module categories of two Morita equivalent algebras are equivalent, and hence it suffices to study the 'simplest' algebra in each Morita equivalence class. In facts, each such equivalence class contains precisely one basic algebra [31] which is the simplest one in the class. Instead of giving their formal definition, I present a general construction of the basic algebras.

\subsection{Quivers and their representations}

A (finite) quiver $Q=\left(Q_{0}, Q_{1}, s, t\right)$ is given by two (finite) sets — the set of 'nodes' $Q_{0}$, and 
the set of 'arrows' $Q_{1}$ - together with two maps $s, t: Q_{1} \rightarrow Q_{0}$ which specify for each arrow $a$ its source and target nodes, $s(a)$ and $t(a)$. A quiver is drawn as an oriented graph, e.g.

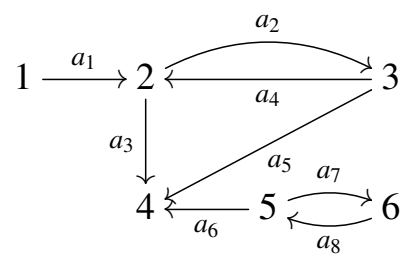

$$
\begin{aligned}
& Q_{0}=\{1,2,3,4,5,6\} \\
& Q_{1}=\left\{a_{1}, a_{2}, a_{3}, a_{4}, a_{5}, a_{6}, a_{7}, a_{8}\right\} .
\end{aligned}
$$

Given a quiver $Q$, we can consider its path algebra $\mathbb{C} Q$. A path in $Q$ of length $n$ is an oriented concatenation of arrows of the form $a_{i_{1}} a_{i_{2}} \cdots a_{i_{n}}$ with $s\left(a_{i_{k}}\right)=t\left(a_{i_{k+1}}\right)$ for all $k=1, \ldots, n-1$. The underlying vector space of the algebra $\mathbb{C} Q$ has a basis consisting of all paths in $Q$ of lenght $n \geq 1$ together with the trivial paths of length zero at each vertex $v, e_{v}$ (called the 'lazy' path at $v$ ). $\mathbb{C} Q$ is an associative algebra, with the product of two paths given by their concatenation, if this makes sense, and zero otherwise; the sum of all lazy paths then is a multiplicative identity. The set $Q_{\geq 1}$ of all paths of lenght $\leq 1$ is a bilateral ideal of $\mathbb{C} Q$, called the arrow ideal. A theorem by Gabriel [31] says that each (finite-dimensional) basic algebra is isomorphic to a quotient algebra of the form $\mathbb{C} Q / I$, where the bilateral ideal $I$ is contained in $\left(Q_{\geq 1}\right)^{2}=Q_{\geq 2}$ and contains $\left(Q_{\geq 1}\right)^{m}=Q_{\geq m}$ for some $m \geq 2$.

Thus it is enough to study the algebras arising from (finite) quivers. The category of modules over such an algebra is equivalent to the category of representations of the quiver (satisfying the relations of $I$ ); a representation $X$ of $Q$ is the assignement of a vector space $X_{v}$ for each vertex $v \in Q_{0}$ and a linear map $X_{a}: X_{s(a)} \rightarrow X_{t(a)}$ for each arrow $a \in Q_{1}$. These linear maps are required to satisfy the relations in $I$.

Given a representation $X$, its dimension vector is defined

$$
\operatorname{dim} X=\left(\operatorname{dim} X_{1}, \operatorname{dim} X_{2}, \cdots, \operatorname{dim} X_{\left|Q_{0}\right|}\right) \in \mathbb{N}^{\left|Q_{0}\right|} .
$$

\subsection{Jacobian algebras}

A particularly important class of basic algebras are the Jacobian ones - originally inspired by work in supersymmetric QFT's, and then understood mathematically. In the original physical framework, one fixes the dimension vector to be $\left(N_{1}, N_{2}, \cdots\right)$, so that the linear maps $X_{a}$ may be seen as $N_{t(a)} \times N_{s(a)}$ matrices, which physicists interpret as Higgs fields in the $\left(\mathbf{N}_{\mathbf{t}(\mathbf{a})}, \overline{\mathbf{N}}_{\mathbf{s}(\mathbf{a})}\right) b i-$ fundamental representation of the 'gauge group' $U\left(N_{t(a)}\right) \times U\left(N_{s(a)}\right)$. In physics such a theory, if supersymmetric, is also equipped with a super-potential $\mathscr{W}$ which is a gauge invariant polynomial in the Higgs fields $X_{a}$; the simplest possibility is a linear combination of traces of the products of the Higgs fields associated to the closed oriented cycles $\alpha^{(\ell)}=a_{1}^{(\ell)} a_{2}^{(\ell)} \cdots a_{m_{\ell}}^{(\ell)}$ in $Q$

$$
\mathscr{W}=\sum_{\ell} \lambda_{\ell} \operatorname{tr}\left(X_{a_{1}^{(\ell)}} X_{a_{2}^{(\ell)}} \cdots X_{a_{m_{\ell}}^{(\ell)}}\right) \quad \lambda_{\ell} \in \mathbb{C}
$$

In a supersymmetric state the Higgs field should satisfy the $F$-flatness condition

$$
\partial_{X_{a}} \mathscr{W}=0 \quad \forall a \in Q_{1}
$$


In mathematics (and on our physical applications) one does not fix $\operatorname{dim} X$, and hence one sees $\mathscr{W}$ more abstractly as a sum of the closed oriented cycles ${ }^{1}$ in $Q$ with complex coefficients $\lambda_{\ell}$. One defines a cyclic derivative $\partial$ of $\mathscr{W}$ (which corresponds to the one in (2.3)) and considers the bilateral ideal $(\partial \mathscr{W})$ generated by the relations $\partial \mathscr{W}=0$. The Jacobian algebra of the pair $(Q, \mathscr{W})$ is then the basic algebra ${ }^{2} \mathbb{C} Q /(\partial \mathscr{W})$.

\section{BPS states in $4 d \mathscr{N}=2$ QFT}

In a $4 \mathrm{D} \mathscr{N}=2$ theory, the BPS particles are those whose mass $M$ and central charge $Z \equiv$ $\varepsilon^{\alpha \beta} \varepsilon_{A B}\left\{Q_{\alpha}^{A}, Q_{\beta}^{A}\right\}$ saturate the Bogomolny bound, $M=|Z|$. A BPS state preserves (i.e. it is invariant under) 4 supercharges. If we consider the world-line theory of a particle, we get a 1D theory with (at most) 4 supercharges; such a theory looks like $\mathscr{N}=1$ supersymmetry in $4 \mathrm{D}$, the chiral superfields taking value in some Kähler manifold. A particle is BPS precisely if it is described by a 1D state which preserves 4 supercharges, that is, if it is a SUSY vacuum of the world-line theory. Typically, the $1 \mathrm{D}$ theory is a quiver theory specified by a quiver $Q$ and a superpotential $\mathscr{W}$. A classical SUSY vacuum is a solution to the equations

$$
D^{a}=0, \quad F_{k}=\partial_{k} \mathscr{W}=0 .
$$

The equation $\partial_{k} \mathscr{W}=0$, precisely corresponds to the Jacobian relations on the Higgs fields. The equation $D^{a}=0$ is a momentum map condition, which by Geometric Invariant Theory is equivalent to the condition of stability, see [32]. To define stability, we have to make more precise the dictionary between physics and quiver representations.

\subsection{From $\mathscr{N}=2$ QFT to RT}

Given a $4 \mathrm{D} \mathscr{N}=2$ QFT we consider the set of all conserved charges (both local and topological) which are linearly realized in a generic point in the Coulomb branch/parameter space ${ }^{3}$. The charges are quantized, and hence take values in some lattice $\Gamma$. In $\Gamma$ there is an integral skewsymmetric form

$$
\begin{aligned}
& \langle\cdot, \cdot\rangle_{\text {Dirac }}: \Gamma \times \Gamma \rightarrow \mathbb{Z}, \\
& \left\langle\gamma, \gamma^{\prime}\right\rangle_{\text {Dirac }}=-\left\langle\gamma^{\prime}, \gamma\right\rangle_{\text {Dirac }}, \gamma, \gamma^{\prime} \in \Gamma,
\end{aligned}
$$

given by the Dirac electric/magnetic pairing. Mutually local charges have zero pairing, and flavor charges (that is, charges which are neither electric nor magnetic) correspond to the radical of the form $\langle\cdot, \cdot\rangle_{\text {Dirac }}$, that is $f$ is a flavor charge iff $\langle f, \gamma\rangle_{\text {Dirac }}=0$ for all $\gamma \in \Gamma$.

The central charge $Z$, being conserved, is a linear combination of the above quantized charges, and hence may be seen as a group homomorphism $Z: \Gamma \rightarrow \mathbb{C}$. A BPS particle of charge $\gamma \in \Gamma$ will

\footnotetext{
${ }^{1}$ In $\mathscr{W}$ we identify cycles which differ by a cyclic permutation of the arrows: $a_{1} a_{2} \cdots a_{m} \equiv a_{2} a_{3} \cdots a_{m} a_{1}$.

${ }^{2}(\partial \mathscr{W}) \subset\left(Q_{1}\right)^{2}$ is automatic for us since all our quivers are 2-acyclic, that is, they do not have oriented cycles of lenght $\leq 2$. The condition $(\partial \mathscr{W}) \supset\left(Q_{1}\right)^{m}$ for some $m$ is also automatic if the algebra is finite-dimensional; otherwise we have the subtleties alluded at the beginning of section 2 .

${ }^{3}$ Tipically the microscopic symmetry algebra is generically broken down to its Cartan subalgebra and our conserved charges commute pairwise. At particular points in the paramenter space we may have non-Abelian enhancements of this Abelian symmetry.
} 


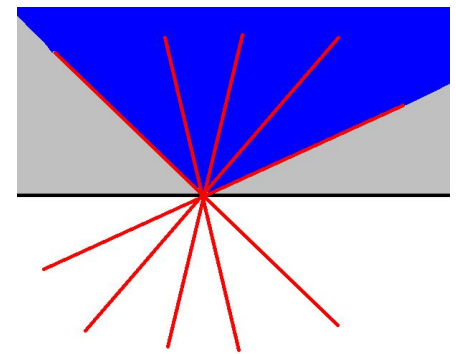

Figure 1: The central charge complex plane. The red vectors are the central charges of BPS states. By convention, we call 'particles' the ones in the upper half-plane, and 'anti-particles' their PCT conjugates in the lower half-plane. This defines the positive cone of 'particles' inside the charge lattice $\Gamma$ (whose image in the $Z$-plane is the blue region in the figure).

then have central charge $Z(\gamma)$ and mass $|Z(\gamma)|$. In most (but not all!) cases the set $\Theta$ of all angles $\theta$ such that there is a BPS particle of charge $\gamma$ with $\theta=\arg Z(\gamma)$ is not everywhere dense in the unit circle. In particular, if the QFTs has 'enough' mass deformation, we may arrange the masses so that there is an angle $\theta_{0}$ which is not in $\bar{\Theta}$. By a change of conventions we may assume $\theta_{0}=0$. Then we have the situation in figure 1 .

We use this figure to distinguish — conventionally — 'particles' vs. 'antiparticles' [25, 26]. The charges of 'particles' define a positive cone $\Gamma_{+}$in $\Gamma$. In a theory of the class described in $[25,26] \Gamma_{+}$is generated by $\operatorname{rank} \Gamma$ generators which we call $e_{i}, i=1,2, \ldots, m=\operatorname{rank} \Gamma$. More precisely, one has

$$
\Gamma=\bigoplus_{i} \mathbb{Z} e_{i}, \quad \Gamma_{+}=\bigoplus_{i} \mathbb{Z}_{+} e_{i}
$$

We shall show that the BPS particle whose central charge is the leftmost (resp. rightmost) one in the upper half-plane has a charge vector equal to a generator $e_{i}$ of the positive cone.

\subsubsection{Quivers and their Seiberg duality (DWZ mutations)}

Given the data $\Gamma,\langle\cdot, \cdot\rangle_{\text {Dirac }}$, and $\Gamma_{+}$, we can construct a candidate quiver $Q$ as follows: the node set $Q_{0}$ is identified with the set of positive generators $\left\{e_{i}\right\}$, and we draw $\left\langle e_{i}, e_{j}\right\rangle_{\text {Dirac }}$ arrows from node $e_{i}$ to node $e_{j}$, where a negative number stands for arrows in the opposite direction $e_{i} \leftarrow e_{j}$. Note that the resulting quivers are always 2-acyclic: all arrows between any two given nodes point in the same directions, and we have no pairs of opposite arrows $\leftrightarrows$ nor loops (i.e. arrows which start and end at the same node). To complete the description of the Jacobian algebra one also needs the superpotential $\mathscr{W}$. Its construction in terms of the $4 \mathrm{D}$ QFT is not easy in general, but it can be done explicitly for large classes of models, including all which have a Lagrangian formulation (e.g. using the techniques of [28]).

The essential observation is that the pair $(Q, \mathscr{W})$ is not unique. Indeed there was some arbitrary choice (as the angle $\theta_{0}$ ) in the definition of the positive cone $\Gamma_{+}$, and different choices lead to different pairs $(Q, \mathscr{W})$ which mus be physically equivalent. From the $1 \mathrm{D}$ QFT on the world-line the ambiguity is related to Seiberg duality [33]: two pairs $(Q, \mathscr{W})$ and $\left(Q^{\prime}, \mathscr{W}^{\prime}\right)$ are related by such a duality. Mathematically, we get a mutation class of quiver with (super)potential as defined by Derksen-Weyman-Zelevinsky [34]. 


\subsubsection{Stability and quantum numbers}

From this construction we see that the positive cone $\Gamma_{+} \equiv\left\{\sum_{i} N_{i} e_{i}\right\}$, is identified with the set of possible dimension vectors $\operatorname{dim} X$ of representations $X$ of the quiver $Q$. Under the identification of the physical charge vectors with dimension vectors, we may define the central charge of a representation of $Q$ ( $\equiv$ a left module of $\mathbb{C} Q /(\partial \mathscr{W}))$ as

$$
Z(X) \equiv Z(\operatorname{dim} X)=\sum_{i} \operatorname{dim} X_{i} Z\left(e_{i}\right) \in \mathscr{H} \Rightarrow 0<\arg Z(X)<\pi .
$$

Both physicists and mathematicians define $X$ to be stable (w.r.t. the stability function $Z(\cdot)$ ) iff for all non-zero proper subrepresentations $Y$ of $X$

$$
\arg Z(Y)<\arg Z(X)
$$

One can show that this is equivalent to the SUSY vacuum condition $D^{a}=0$ [26]. Therefore, if $X$ is a stable object in $\bmod \mathbb{C} Q /(\partial \mathscr{W})$, the corresponding $1 \mathrm{D}$ Higgs field configuration satisfies both the $D$ - and $F$-flatness condition and hence is a classical supersymmetric vacuum, and thus a BPS state of the 4D theory with charges (quantum numbers) $\operatorname{dim} X$. More precisely, those classical vacua come, in general, in continuous families of gauge-inequivalent ones. In the mathematical language, they are continuous families of pairwise non-isomorphic stable representations. Physically, the family coordinates are 'global coordinates' which we should quantize; their quantization produces the last quantum number of the particle, namely its spin. A (complex) dimension $d$ family produces a BPS supermultiplet whose highest spin is

$$
\text { higher } \operatorname{spin}=\frac{d+1}{2} \text {. }
$$

So $d=0$ ( $X$ rigid) corresponds to hypermultiplets, and $d=1$ to vector multiplets.

In conclusion, for a quiver $\mathscr{N}=2$ theory, to get the full BPS spectrum (masses and quantum numbers of all BPS particles) one has to determine the set of stable representations of a certain associative algebra. This is the canonical problem of Representation Theory.

\subsubsection{Chambers, wall-crossing, derived category}

Of course, stability depends on $Z(\cdot)$, that is on the $\operatorname{rank} \Gamma$ complex numbers $Z\left(e_{i}\right)$, which are functions of all the physical parameters of the theory; changing the parameters may lead to different BPS spectra. These correspond to different BPS chambers, and the spectra on two different chambers are related by the Kontesevich-Soibelman wall-crossing formula [35]-[43].

As we already mentioned, our distinction of the states into 'particles' and 'antiparticles' is rather unphysical. A more satisfactory, totally invariant formulation exists: one replaces the Abelian category of modules of the Jacobian algebra by the corresponding derived category

$$
D(\bmod \mathbb{C} Q /(\partial \mathscr{W}))
$$

There is a more intrinsic definition of stability at the derived category level, see [44], which reduces to the above one in our more elementary treatment. 


\subsubsection{Bricks and stability}

One shows [32] that a necessary condition for a module $X$ to be stable for some choice of the central charge ${ }^{4} Z(\cdot)$ is that $X$ is a brick, i.e. that it has a trivial endomorphism ring, End $X=\mathbb{C}$. We claim that the brick condition is also sufficient [28]. To show this, we consider the forgetful functor $F: \bmod \mathbb{C} Q /(\partial \mathscr{W}) \rightarrow \bmod \mathbb{C} Q$ defined by forgetting that our modules satisfy the the relations $\partial \mathscr{W}=0 . X$ and $F(X)$ have the same central charge, and $X$ is a brick iff $F(X)$ is a brick. $Y$ is a submodule of $X$, implies that $F(Y)$ is a submodule of $F(X)$. Then, if $F(X)$ is stable for some choice of $Z(\cdot), X$ is also stable. We consider the family of central charges $Z_{t}(\cdot)$

$$
Z_{t}(\cdot)=Z_{0}(\cdot)+t\left(\langle\cdot, \operatorname{dim} F(X)\rangle_{E}-\langle\operatorname{dim} F(X), \cdot\rangle_{E}\right), \quad t \rightarrow+\infty .
$$

where $\langle\cdot, \cdot\rangle_{E}$ is the Euler form of the quiver $Q$ and

$$
\left\langle\gamma, \gamma^{\prime}\right\rangle_{\text {Dirac }}=\left\langle\gamma^{\prime}, \gamma\right\rangle_{E}-\left\langle\gamma, \gamma^{\prime}\right\rangle_{E}
$$

The fact that $F(X)$, assumed to be a brick, is stable as $t \rightarrow+\infty$ is equivalent to the characterization of the dimension vectors of bricks, see [45]. Notice that as $t \rightarrow+\infty$, the states which remain light are precisely those mutually local with respect to $X$.

\subsubsection{First consequences and remarks}

A simple representation $S$ has no non-trivial subrepresentions, and the stability requirement (3.5) is empty, hence automatically satisfied. If the Jacobian algebra is finite-dimensional, the only simple representations are the $S_{i}, i \in Q_{0}$, with $\left(\operatorname{dim} S_{i}\right)_{j}=\delta_{i j}$, that is, the ones whose dimension vector is a generator $e_{i}$ of the positive cone $\Gamma_{+}$. These simple representations are rigid, hence correspond to hypermultiplets which are stable in all the BPS chambers covered by the particular quiver in the mutation class. From this observation we learn a few general results:

1. Not all $\mathscr{N}=2$ theories may have a quiver formulation. A necessary condition for the existence of a finite quiver is that the closure of the BPS phase set $\bar{\Theta} \neq S^{1}$; moreover, the phase of each boundary component of $\bar{\Theta}$, which correspond to the generators of minimal/maximal $\arg Z\left(e_{i}\right)$, should correspond to a single BPS hypermultiplet, and not to a higher spin BPS multiplet or to many distinct hypermultiplets. This is not always true: for instance, if we consider $\mathscr{N}=4$, seen as a particular instance of $\mathscr{N}=2$, the particles organize themselves in $\mathscr{N}=4$ supermultiplets which decompose in a plurality of $\mathscr{N}=2$ ones: this is not consistent with a quiver. However, breaking $\mathscr{N}=4 \rightarrow \mathscr{N}=2$ by giving a mass to the adjoint hypermultiplet we get a quiver theory;

2. In all BPS chambers, a quiver $\mathscr{N}=2$ theories must have a BPS spectrum which contains at least $\operatorname{rank} \Gamma$ hypermultiplets (with linearly independent charges) corresponding to the $S_{i}$ 's. We may say that a chamber with just this BPS spectrum - $\operatorname{rank} \Gamma$ hypermultiplets - is a minimal chamber. A natural question is: 'Which $4 d \mathscr{N}=2$ theories do have a minimal chamber?' The answer is given in the next subsection.

\footnotetext{
${ }^{4}$ Such that $\operatorname{Im} Z\left(e_{i}\right)>0$ for all $i$, to be consistent with our splitting of 'particles' vs. 'antiparticles'.
} 


\subsection{Not all pairs $(Q, \mathscr{W})$ correspond to consistent QFTs}

One may ask whether all mathematically consistent pairs $(Q, \mathscr{W})$ correspond to consistent QFT. The answer is no. In [23] a (conjectural) characterization was given in the physical language. That characterization leads to a necessary condition which is easy to state in RT terms: there should exist a finite-dimensional basic algebra $\mathscr{B}$, having global dimension at most 2 , with the properties

1. $K_{0}(\bmod \mathscr{B}) \simeq \Gamma$ and the minimal Jacobian algebra containing $\mathscr{B}$ (as a subalgebra) is $\mathbb{C} Q /(\partial \mathscr{W})$;

2. the Coxeter element of $\mathscr{B}$ has spectral radius 1 and Jordan blocks of size at most 3 .

For the particular case of hereditary algebras, that is, for pairs of the form $(Q, 0)$ (equivalently, algebras of global dimension at most 1) - which contain all cases in which $Q$ has no closed oriented loop - a theorem of Ringel [46] gives the full classification of the algebras satisfying the necessary condition of [23]. They are the path algebras of quivers which are orientations of either the $A D E$ Dynkin graphs of the affine (Euclidean) Dynkin graphs $\widehat{A} \widehat{D} \widehat{E}$, in the case $\widehat{A}_{n}$ the orientation producing a closed oriented loop must be excluded (this quiver also corresponds to a consistent QFT, but only if equipped with a superpotential equal to the oriented cycle; the pair $\left(\left.\widehat{A}_{n}\right|_{\text {cycl. }}, a_{1} a_{2} \cdots a_{n+1}\right)$ is then Seiberg equivalent to the one associated to the $D_{n+1}$ Dynkin quiver).

Except for the $\widehat{A}_{n}$ case, all possible orientations of these Dynkin quivers are Seiberg duality equivalent, and hence correspond to the same $\mathscr{N}=2$ theory; for $\widehat{A}_{n}$ two orientations are equivalent iff they have the same number of arrows in the clockwise direction, we write the class as $\widehat{A}(p, q)$ with $p \geq q \geq 1$, where $p$ and $q$ are, respectively the number of arrows in the clockwise and anticlockwise direction. This corresponds to the fact that for all finite-dimensional or affine KacMoody algebras whose Dynkin graph is a tree all Coxeter elements belong to the same conjugacy class, while for $\widehat{A}$ the conjugacy classes depend on an extra datum which may be identified with the number of positively oriented links in a orientation of the graph.

We shall see the physics of these Dynkin and affine Dynkin models below. Here we just state a consequence of Ringel's theorem

Fact. The class of models with pair $(Q, 0)$, where $Q$ is an acyclic orientation of a finitedimensional or affine ADE Dynkin graph, coincides with the class of $\mathscr{N}=2$ models having a minimal BPS chamber.

\subsection{Vector-less theories and Gabriel theorem}

Theories whose BPD spectrum consists of finitely many hypermultiplets in all chambers are called vector-less theories. One shows that this class contains just the theories associated to the Dynkin quivers (however, in their mutation classes we have many pairs $(Q, \mathscr{W})$ with $Q$ not an orientation of a Dynkin graph). These $\mathscr{N}=2$ theories have an $A D E$ classification and are called Argyres-Douglas theories. They have no Lagrangian formulation (expect for $A_{1}$ and $D_{2}$ which are free) but may be constructed e.g. using the $6 \mathrm{~d}(2,0)$ SCFT or Type IIB superstring. We may limit to consider quivers in the Dynkin form (they cover everything); then the BPS states are described by Gabriel theorem [31]. There is a maximal chamber where all the bricks of the hereditary algebra $\mathbb{C} Q$ are stable BPS states. By Gabriel theorem they are precisely the positive roots of the 
corresponding algebra as

$$
\operatorname{dim} X=\sum_{i} n_{i} e_{i} \equiv \sum_{i} n_{i} \alpha_{i}, \quad \Gamma \equiv \Gamma_{\text {root }} \text { via } e_{i} \leftrightarrow \alpha_{i} \text { (simple roots) }
$$

The minimal chamber has only the BPS states associated with the simple roots, and all other chambers are in between these two.

\subsection{Complete theories}

The theories whose quiver mutation class is finite (with the exception of the $k$-Kronecker quivers with $k \geq 3$ ) are called complete. They have many nice properties

- all choices of the central charge $Z(\cdot)$ may be physically realized as a UV complete QFT;

- in all chambers the BPS states have at most spin 1;

- the WKB BPS spectrum is exact;

- the gauge group is $S U(2)^{k}$ in all $S$-duality frames.

Mathematically, the corresponding Jacobian algebras are tame. These $\mathscr{N}=2$ models have been fully classified [24]: they consist on all generalized Gaiotto $A_{1}$ theories [19] (where 'generalized' means that irregular punctures of any order are allowed) together with 11 exceptional models.

\subsection{Lie algebraic aspects}

The simplest class of complete gauge theories are those associated to an affine $A D E$ quiver. The situation is totally similar to the one described by Gabriel theorem for the finite-dimensional $A D E$ Dynkin quivers, and the extension of the Gabriel theorem to this case is given by a theorem by Kac [47].

The new ingredient is that in the affine case we have two kinds of roots: real roots with ${ }^{5}$ $(\alpha, \alpha)=2$ and imaginary roots with $(\alpha, \alpha)=0$. We have a simple formula for the dimension of the moduli space

$$
\operatorname{dim} \mathscr{M}(X)=1-\frac{1}{2}(\operatorname{dim} X, \operatorname{dim} X) .
$$

Since $(\cdot, \cdot)$ is positive semi-definite, we see that from eqn.(3.6) that a representation $X$ which corresponds to an hypermultiplet has a dimension vector (charge) which is a real root under the identification $\Gamma \simeq \Gamma_{\text {root }}$, while if it is a vector-multiplet $\operatorname{dim} X$ is a imaginary root, and no spin $>1$ is possible.

However, a state may be stable only if the corresponding representation is a brick. An indecomposable with dimension $\delta$, the minimal imaginary root, is always a brick, which we may identity with the $W$-boson in the Higgs breaking $S U(2) \rightarrow U(1)$. This gives us the possibility of defining the magnetic charge

$$
\begin{aligned}
& m(\cdot): \Gamma \rightarrow \mathbb{Z} \\
& m(X)=\frac{1}{2}\langle\operatorname{dim} X, \delta\rangle_{\text {Dirac }}
\end{aligned}
$$

\footnotetext{
$5(\cdot, \cdot)$ is the quadratic form on the root lattice given by the Cartan matrix.
} 
a quantity which is known in mathematics as the Dlab-Ringel defect [48]. Then

Theorem [49]. An indecomposable representation $X$ of an acyclic affine quiver is a brick iff one of the following hold:

1. $\operatorname{dim} X$ is a root and $m(X) \neq 0$;

2. $\operatorname{dim} X$ is a root $m(x)=0$ and $\operatorname{dim} X \leq \delta$.

In the maximal BPS chamber - corresponding to weak coupling $g_{\mathrm{YM}} \sim 0$ - all bricks correspond to stable BPS particles (in the minimal chamber, corresponding to strong coupling ${ }^{6}$, we have only the simple rep. $S_{i}$ ). Hence, in the maximal chamber, we get two infinite towers of hypermultiplets dyons, corresponding to the real roots with $m(X)>0$ and $m(X)<0$, and finitely many states of zero magnetic charge, with just one vector multiplet, corresponding to the unique $W$-boson.

This shows that an affine quiver corresponds to $S U(2)$ SYM coupled to matter in the fundamental rep. The dyons have masses of $O\left(1 / g^{2}\right)$, and as $g \rightarrow 0$ we get the 'perturbative spectrum'. In the decoupling limit the matter separates from the SYM sector. One sees that it corresponds to a bunch of Argyres-Douglas systems of type- $D_{r}$, which have a global $S U(2)$ symmetry which may be gauged. In facts, the affine quiver correspond to $S U(2)$ SYM coupled to Argyres-Douglas matter of type $D_{r}$ ( $D_{2}$ being the free hypermultiplet doublet) such that the beta-function of the YM coupling remains negative, so that the theory is asymptotically free (where the coupling of a $D_{r}$ Argyres-Douglas system reduces the leading coefficient of $\beta$ by $2(1-1 / r)[24,28])$.

\subsubsection{SCFT: $S U(2)$ SQCD with $N_{f}=4$}

In the language above this corresponds to $S U(2)$ SYM coupled to four copies of the $D_{2}$ Argyres-Douglas. The coefficient of $\beta$ is $4-4 \times 1=0$ and the model is conformal and hence it cannot be described by any affine Dynkin quiver. Does the relation between the Representation Theory of Lie algebras and the BPS spectra break down?

Certainly not. One has simply to enlarge the set of relevant Lie algebras. This conformal model corresponds to the toroidal Lie algebra $D_{4}^{(1,1)}[50,51]$. There is a natural action of the modular group $S L(2, \mathbb{Z})$ on the root lattice of this algebra which preserves the isotropic imaginary roots. Physically this automorphism of the toroidal root lattice is the $S$-duality group, see [28].

\subsubsection{Conformal $S U(2)$ quiver theories}

The above result generalizes to all $S U(2)$ quiver SCFTs. These are the $A_{1}$ Gaiotto theories

- defined by genus-zero surface with $p \geq 4$ punctures if the quiver is a A-type Dynkin graph. The gauge group has $(p-3) S U(2)$ factors, represented by circles in figure 2 A) (boxes represent flavor $S U(2)$ 's associated to fundamental hypers and edges bifundamental ones);

- genus-one surfaces with $p \geq 1$ punctures if the underlying graph of the quiver is an affine $A$-type graph. The gauge group is $S U(2)^{p}$; each $S U(2)$ factor is represented by a circle in figure $2 \mathrm{~B}$ );

${ }^{6}$ For affine $D, E$ there exists also submaximal weak coupling chambers in which the dyons with magnetic charge $|m|>1$ have being decayed to fundamental dyons with $|m|=1$. 
A)

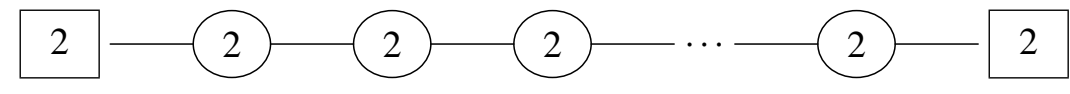

B)

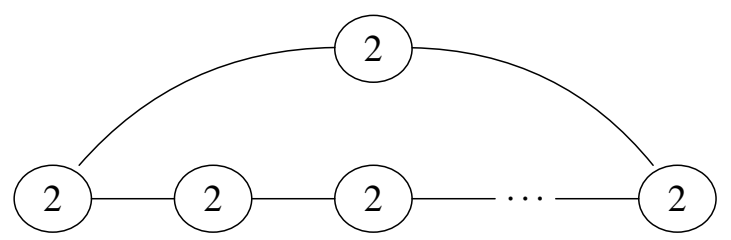

Figure 2: A) the Dynkin quiver corresponding to the Gaiotto theory on the genus-zero surface with $p$ punctures; B) the affine quiver corresponding to the Gaiotto theory on the genus-one surface with $p$ punctures.

Note that the first models in these classes correspond, respectively, to $S U(2)$ SQCD with $N_{f}=4(\S .3 .5 .1)$ and the $S U(2) \mathscr{N}=2^{*}$ theory.

One can show that the spectrum of the first class of theories is captures by the extended affine Lie algebra [52] of type $D_{2(p-2)}$ and nullity $p-2$

$$
D_{2(p-2)}^{(1,1,1, \cdots, 1)}, \quad(\#\{1 \text { 's }\}=p-2)
$$

while the second one by the extended affine Lie algebra of type $A_{2 p-1}$ and nullity $p+1$

$$
A_{2 p-1)}^{(1,1,1, \cdots, 1)}, \quad(\#\{1 \text { 's }\}=p+1)
$$

Note that for $p=4$ (resp. $p=1$ ) we get back the 'classical' result of $\S .3 .5 .1$ (the know result for $\left.\mathscr{N}=2^{*}\right)$.

\section{References}

[1] N. Seiberg and E. Witten, Electric - magnetic duality, monopole condensation, and confinement in N=2 supersymmetric Yang-Mills theory, Nucl. Phys. B426 (1994) 19-52, arXiv:hep-th/9407087 [hep-th].

[2] N. Seiberg and E. Witten, Monopoles, duality and chiral symmetry breaking in N=2 supersymmetric QCD, Nucl. Phys. B431 (1994) 484-550, arXiv: hep-th/9408099 [hep-th].

[3] F. Denef, Supergravity flows and D-brane stability, JHEP 0008 (2000) 050, arXiv:hep-th/0005049 [hep-th].

[4] F. Denef and G. W. Moore, Split states, entropy enigmas, holes and halos, arXiv:hep-th/0702146 [hep-th].

[5] M. R. Douglas and G. W. Moore, D-branes, Quivers, and ALE Instantons, arXiv:hep-th/9603167.

[6] D.-E. Diaconescu and J. Gomis, Fractional branes and boundary states in orbifold theories, JHEP 0010 (2000) 001, arXiv: hep-th/9906242 [hep-th] .

[7] M. R. Douglas, B. Fiol, and C. Römelsberger, Stability and BPS branes, JHEP 0509 (2005) 006, arXiv:hep-th/0002037 [hep-th]. 
[8] M. R. Douglas, B. Fiol, and C. Römelsberger, The Spectrum of BPS branes on a noncompact Calabi-Yau, JHEP 0509 (2005) 057, arXiv: hep-th/0003263 [hep-th] .

[9] B. Fiol and M. Marino, BPS states and algebras from quivers, JHEP 0007 (2000) 031 , arXiv:hep-th/0006189 [hep-th].

[10] B. Fiol, The BPS spectrum of $N=2 S U(N) S Y M$ and parton branes, arXiv : hep-th/0012079.

[11] F. Denef, Quantum quivers and Hall / hole halos, JHEP 0210 (2002) 023, arXiv:hep-th/0206072 [hep-th].

[12] B. Feng, A. Hanany, Y. H. He, and A. Iqbal, Quiver theories, soliton spectra and Picard-Lefschetz transformations, JHEP 0302 (2003) 056, arXiv: hep-th/0206152 [hep-th] .

[13] B. Feng, A. Hanany, and Y.-H. He, D-brane gauge theories from toric singularities and toric duality, Nucl.Phys. B595 (2001) 165-200, arXiv: hep-th/0003085 [hep-th] .

[14] B. Feng, A. Hanany, and Y.-H. He, Phase structure of D-brane gauge theories and toric duality, JHEP 0108 (2001) 040, arXiv: hep-th/0104259 [hep-th] .

[15] A. Hanany and K. D. Kennaway, Dimer models and toric diagrams, arXiv: hep-th/ 0503149 [hep-th].

[16] S. Franco, A. Hanany, K. D. Kennaway, D. Vegh, and B. Wecht, Brane dimers and quiver gauge theories, JHEP 0601 (2006) 096, arXiv: hep-th/0504110 [hep-th] .

[17] B. Feng, Y.-H. He, K. D. Kennaway, and C. Vafa, Dimer models from mirror symmetry and quivering amoebae, Adv.Theor.Math.Phys. 12 (2008) 3, arXiv:hep-th/0511287 [hep-th] .

[18] A.D. Shapere and C. Vafa, BPS structure of Argyres-Douglas superconformal theories, hep-th/9910182

[19] D. Gaiotto, N=2 dualities, arXiv:0904.2715 [hep-th].

[20] D. Gaiotto, G. W. Moore, and A. Neitzke, Wall-crossing, Hitchin Systems, and the WKB Approximation, arXiv:0907.3987 [hep-th].

[21] D. Gaiotto, G. W. Moore, and A. Neitzke, Framed BPS States, arXiv:1006.0146 [hep-th].

[22] D. Gaiotto, G. W. Moore, and A. Neitzke, Spectral networks, arXiv:1204.4824 [hep-th].

[23] S. Cecotti, A. Neitzke, and C. Vafa, R-Twisting and 4d/2d Correspondences, arXiv: 1006.3435 [hep-th].

[24] S. Cecotti and C. Vafa, Classification of complete $N=2$ supersymmetric theories in 4 dimensions, arXiv:1103.5832 [hep-th].

[25] M. Alim, S. Cecotti, C. Cordova, S. Espahbodi, A. Rastogi, and C. Vafa, BPS Quivers and Spectra of Complete N=2 Quantum Field Theories, arXiv:1109.4941 [hep-th] .

[26] M. Alim, S. Cecotti, C. Cordova, S. Espahbodi, A. Rastogi, and C. Vafa, $N=2$ Quantum Field Theories and their BPS Quivers, arXiv:1112.3984 [hep-th] .

[27] S. Cecotti and M. Del Zotto, On Arnold's 14 'exceptional' $\mathscr{N}=2$ superconformal gauge theories, JHEP 1110 (2011) 099, arXiv: 1107.5747 [hep-th] .

[28] S. Cecotti, Categorical tinkertoys for $N=2$ gauge theories, arXiv:1203.6743 [hep-th].

[29] S. Cecotti and M. Del Zotto, Half-Hypers and Quivers, JHEP 09 (2012) 135, arXiv: 1207.2275 $[$ hep-th]. 
[30] S. Cecotti and M. Del Zotto, $4 d N=2$ Gauge Theories and Quivers: the Non-Simply Laced Case, JHEP 10 (2012) 190, arXiv:1207.7205 [hep-th].

[31] I. Assem, D. Simson and A. Skowronski, Elements of the Representation Theory of Associative Algebras. 1: Techniques of Representation Theory, LONDON MATHEMATICAL SOCIETy STUdENT Texts 65, Cambridge Univerty Press, Cambridge, (2006).

[32] A.D. King, Moduli of representations of finite-dimensional algebras, Quart. J. Math. Oxford Ser.(2) 45 (1994), 515-530.

[33] N. Seiberg, Electric - magnetic duality in supersymmetric nonAbelian gauge theories, Nucl.Phys. B435 (1995) 129-146, arXiv: hep-th/9411149 [hep-th].

[34] H. Derksen, J. Wyman, and A. Zelevinsky, Quivers with potentials and their representations I: Mutations, Selecta Mathematica 14 (2008) 59-119.

[35] M. Kontsevich and Y. Soibelman, Stability structures, motivic Donaldson-Thomas invariants and cluster transformations, ArXiv e-prints (Nov., 2008), arXiv:0811.2435 [math.AG] .

[36] T. Dimofte and S. Gukov, Refined, Motivic, and Quantum, Lett. Math. Phys. 91 (2010) 1, arXiv:0904.1420 [hep-th].

[37] S. Cecotti and C. Vafa, BPS Wall Crossing and Topological Strings, arXiv:0910.2615 [hep-th].

[38] T. Dimofte, S. Gukov, and Y. Soibelman, Quantum Wall Crossing in N=2 Gauge Theories, Lett.Math.Phys. 95 (2011) 1-25, arXiv:0912.1346 [hep-th].

[39] D. Gaiotto, G. W. Moore, and A. Neitzke, Framed BPS States, arXiv:1006.0146 [hep-th].

[40] E. Andriyash, F. Denef, D. L. Jafferis, and G. W. Moore, Wall-crossing from supersymmetric galaxies, arXiv:1008.0030 [hep-th].

[41] E. Andriyash, F. Denef, D. L. Jafferis, and G. W. Moore, Bound state transformation walls, arXiv:1008.3555 [hep-th].

[42] J. Manschot, B. Pioline, and A. Sen, Wall Crossing from Boltzmann Black Hole Halos, JHEP 1107 (2011) 059, arXiv:1011.1258 [hep-th].

[43] D. Gaiotto, G. W. Moore, and A. Neitzke, Wall-Crossing in Coupled 2d-4d Systems, arXiv:1103.2598 [hep-th].

[44] T. Bridgeland, Spaces of stability conditions, arXiv:math/0611510.

[45] A. Schofield, General representations of quivers, Proc. London Math. Soc. (3) 65 (1992) $46-64$.

[46] C.M. Ringel, The spectral radius of the Coxeter transformations for a generalized Cartan Matrix,' Math. Ann. 300 331-339 (1994).

[47] V.G. Kac Infinite Root Systems, Representations of Graphs and Invariant Theory, Inv. Math. 56 (1980) 56-92.

[48] R. Stekolshchik, Notes on Coxeter Transformations and the McKay Correspondence, SPRINGER Monographs In Mathematics, Springer, Berlin, (2008).

[49] W. Crawley-Boevey, Lectures on Representations of Quivers, available on line at http://www1.maths.leeds.ac.uk/ pmtwc/quivlecs.pdf.

[50] K. Saito, Extended affine root systems.I. Coxeter transformations, Publ. Res. Inst. Math. Sci. 21 (1985) 75-179. 
[51] R.V. Moody, S. Eswara Rao and T. Yokonuma, Toroidal Lie algebras and Vertex representations, Geom. Dedicata 35 (1990) 283-307.

[52] E. Neher, Extended affine Lie algebras and other generalizations of affine Lie algebras - A survery, arXiv:0805.3460 [math.RA]. 